\title{
On Some Iterative Methods with Memory and High Efficiency Index for Solving Nonlinear Equations
}

\author{
Tahereh Eftekhari \\ Faculty of Mathematics, University of Sistan and Baluchestan, Zahedan 987-98155, Iran \\ Correspondence should be addressed to Tahereh Eftekhari; t.eftekhari2009@gmail.com
}

Received 25 December 2013; Accepted 10 March 2014; Published 6 April 2014

Academic Editor: Fawang Liu

Copyright (C) 2014 Tahereh Eftekhari. This is an open access article distributed under the Creative Commons Attribution License, which permits unrestricted use, distribution, and reproduction in any medium, provided the original work is properly cited.

Based on iterative methods without memory of eighth-order convergence proposed by Thukral (2012), some iterative methods with memory and high efficiency index are presented. We show that the order of convergence is increased without any additional function evaluations. Numerical comparisons are made to show the performance of the presented methods.

\section{Introduction}

Multipoint iterative methods for solving nonlinear equations are of great practical importance since they overcome theoretical limits of one-point methods concerning the convergence order and computational efficiency. The main goal and motivation in constructing root-solvers is to achieve as high as possible convergence order consuming as small as possible function evaluations. Let $f$ be a sufficiently smooth function of single variable in some neighborhood $D$ of $\alpha$, where $\alpha$ satisfies $f(\alpha)=0$. Traub [1] considered the iterative function of order two:

$$
x_{n+1}=x_{n}-\frac{\beta f\left(x_{n}\right)^{2}}{f\left(x_{n}+\beta f\left(x_{n}\right)\right)-f\left(x_{n}\right)},
$$

where $\beta \neq 0$ is a real constant. The choice $\beta=1$ produces the well-known Steffensen method [2]. To improve the local order of convergence, many modified methods have been proposed in the open literature; see [3-11] and references therein. Thukral developed a scheme of optimal order of convergence eight [12], constructing a weight function as well in the following form:

$$
\begin{aligned}
& \phi_{1}=\left(1-\frac{f\left(y_{n}\right)}{f\left(w_{n}\right)}\right)^{-1}, \\
& \phi_{2}=1+\frac{f\left(y_{n}\right)}{f\left(w_{n}\right)}+\left(\frac{f\left(y_{n}\right)}{f\left(w_{n}\right)}\right)^{2},
\end{aligned}
$$

$$
\begin{aligned}
\phi_{3}= & \frac{f\left[x_{n}, w_{n}\right]}{f\left[w_{n}, y_{n}\right]}, \\
w_{n}= & x_{n}+\beta f\left(x_{n}\right) \\
y_{n}= & x_{n}-\frac{\beta f\left(x_{n}\right)^{2}}{f\left(w_{n}\right)-f\left(x_{n}\right)}, \\
z_{n}= & y_{n}-\phi_{k}\left(\frac{f\left(y_{n}\right)}{f\left[x_{n}, y_{n}\right]}\right), \quad k=1,2,3 \\
x_{n+1}= & z_{n}-\left(1-\frac{f\left(z_{n}\right)}{f\left(w_{n}\right)}\right)^{-1} \\
& \times\left(1-\frac{f\left(y_{n}\right)^{3}}{f\left(w_{n}\right)^{2} f\left(x_{n}\right)}\right)\left(\frac{f\left[x_{n}, y_{n}\right] f\left(z_{n}\right)}{f\left[y_{n}, z_{n}\right] f\left[x_{n}, z_{n}\right]}\right),
\end{aligned}
$$

where $\beta \neq 0$ is a real constant. These methods belong to the class of methods without memory. In this paper, we use optimal multipoint method without memory by Thukral as the base for constructing considerably faster methods employing information from the current and previous iteration without any additional evaluations of the function. Following Traub's classification (see [1, pp. 8-9]), this class of root-finders is called methods with memory. Our main goal is to present some multipoint methods with high efficiency 
index to find the approximation of the root $\alpha$ of the nonlinear equation $f(x)=0$. The acceleration of convergence rate is attained by suitable variation of one free parameter in each iterative step. This self-accelerating parameter is calculated using information from the current and previous iteration by applying Newton's interpolating polynomials. Since considerable acceleration of convergence is obtained without additional function evaluations, the computational efficiency of improved multipoint methods is significantly increased. The efficacy of the methods is tested on a number of numerical examples.

\section{Multipoint Methods with Memory}

In [13], Sharma et al. have presented three methods through the following forms of $\beta_{n}$ :

$$
\begin{aligned}
& \beta_{n}=-\frac{1}{\bar{f}^{\prime}(\alpha)}=-\frac{1}{N_{3}^{\prime}\left(x_{n}\right)}, \\
& \beta_{n}=-\frac{1}{\bar{f}^{\prime}(\alpha)}=-\frac{1}{\bar{N}_{3}^{\prime}\left(x_{n}\right)}, \\
& \beta_{n}=-\frac{1}{\bar{f}^{\prime}(\alpha)}=-\frac{1}{N_{4}^{\prime}\left(x_{n}\right)},
\end{aligned}
$$

for $n=1,2, \ldots$, where $\bar{f}^{\prime}(\alpha)$ denotes an approximation to $f^{\prime}(\alpha)$, and

$$
\begin{aligned}
& N_{3}(t)=N_{3}\left(t ; x_{n}, z_{n-1}, y_{n-1}, x_{n-1}\right), \\
& \bar{N}_{3}(t)=\bar{N}_{3}\left(t ; x_{n}, z_{n-1}, y_{n-1}, w_{n-1}\right)
\end{aligned}
$$

are Newton's interpolatory polynomials of the third degree, set through four available approximations:

$$
\left(x_{n}, z_{n-1}, y_{n-1}, x_{n-1}\right), \quad\left(x_{n}, z_{n-1}, y_{n-1}, w_{n-1}\right),
$$

respectively, and $N_{4}(t)=N_{4}\left(t ; x_{n}, z_{n-1}, y_{n-1}, w_{n-1}, x_{n-1}\right)$ is Newton's interpolatory polynomial of the fourth degree, set through five available $\left(x_{n}, z_{n-1}, y_{n-1}, w_{n-1}, x_{n-1}\right)$ approximations. The derivatives are as follows:

$$
\begin{aligned}
N_{3}^{\prime}\left(x_{n}\right)=\left[\frac{d}{d t} N_{3}(t)\right]_{t=x_{n}} \\
=\left[\frac { d } { d t } \left(f\left(x_{n}\right)+f\left[x_{n}, z_{n-1}\right]\left(t-x_{n}\right)\right.\right. \\
\quad+f\left[x_{n}, z_{n-1}, y_{n-1}\right]\left(t-x_{n}\right)\left(t-z_{n-1}\right) \\
\quad+f\left[x_{n}, z_{n-1}, y_{n-1}, x_{n-1}\right] \\
\left.\left.\quad \times\left(t-x_{n}\right)\left(t-z_{n-1}\right)\left(t-y_{n-1}\right)\right)\right]_{t=x_{n}} \\
=f\left[x_{n}, z_{n-1}\right]+f\left[x_{n}, z_{n-1}, y_{n-1}\right]\left(x_{n}-z_{n-1}\right) \\
+f\left[x_{n}, z_{n-1}, y_{n-1}, x_{n-1}\right]\left(x_{n}-z_{n-1}\right)\left(x_{n}-y_{n-1}\right),
\end{aligned}
$$

$$
\begin{aligned}
& \bar{N}_{3}^{\prime}\left(x_{n}\right)=\left[\frac{d}{d t} \bar{N}_{3}(t)\right]_{t=x_{n}} \\
& =\left[\frac { d } { d t } \left(f\left(x_{n}\right)+f\left[x_{n}, z_{n-1}\right]\left(t-x_{n}\right)\right.\right. \\
& +f\left[x_{n}, z_{n-1}, y_{n-1}\right]\left(t-x_{n}\right)\left(t-z_{n-1}\right) \\
& +f\left[x_{n}, z_{n-1}, y_{n-1}, w_{n-1}\right] \\
& \left.\left.\times\left(t-x_{n}\right)\left(t-z_{n-1}\right)\left(t-y_{n-1}\right)\right)\right]_{t=x_{n}} \\
& =f\left[x_{n}, z_{n-1}\right]+f\left[x_{n}, z_{n-1}, y_{n-1}\right]\left(x_{n}-z_{n-1}\right) \\
& +f\left[x_{n}, z_{n-1}, y_{n-1}, w_{n-1}\right]\left(x_{n}-z_{n-1}\right)\left(x_{n}-y_{n-1}\right), \\
& N_{4}^{\prime}\left(x_{n}\right)=\left[\frac{d}{d t} N_{4}(t)\right]_{t=x_{n}} \\
& =\left[\frac { d } { d t } \left(f\left(x_{n}\right)+f\left[x_{n}, z_{n-1}\right]\left(t-x_{n}\right)\right.\right. \\
& +f\left[x_{n}, z_{n-1}, y_{n-1}\right]\left(t-x_{n}\right)\left(t-z_{n-1}\right) \\
& +f\left[x_{n}, z_{n-1}, y_{n-1}, w_{n-1}\right] \\
& \times\left(t-x_{n}\right)\left(t-z_{n-1}\right)\left(t-y_{n-1}\right) \\
& +f\left[x_{n}, z_{n-1}, y_{n-1}, w_{n-1}, x_{n-1}\right]\left(t-x_{n}\right) \\
& \left.\left.\times\left(t-z_{n-1}\right)\left(t-y_{n-1}\right)\left(t-w_{n-1}\right)\right)\right]_{t=x_{n}} \\
& =f\left[x_{n}, z_{n-1}\right]+f\left[x_{n}, z_{n-1}, y_{n-1}\right]\left(x_{n}-z_{n-1}\right) \\
& +f\left[x_{n}, z_{n-1}, y_{n-1}, w_{n-1}\right]\left(x_{n}-z_{n-1}\right)\left(x_{n}-y_{n-1}\right) \\
& +f\left[x_{n}, z_{n-1}, y_{n-1}, w_{n-1}, x_{n-1}\right] \\
& \times\left(x_{n}-z_{n-1}\right)\left(x_{n}-y_{n-1}\right)\left(x_{n}-w_{n-1}\right) .
\end{aligned}
$$

Now, we replace constant parameters $\beta$ in the iterative formula (5) by the varying $\beta_{n}$ defined by (6), (7), and (8). Then, the multipoint methods with memory, following from (5), become

$$
\begin{aligned}
& w_{n}=x_{n}+\beta_{n} f\left(x_{n}\right), \\
& y_{n}=x_{n}-\frac{\beta_{n} f\left(x_{n}\right)^{2}}{f\left(w_{n}\right)-f\left(x_{n}\right)}, \\
& z_{n}=y_{n}-\phi_{k}\left(\frac{f\left(y_{n}\right)}{f\left[x_{n}, y_{n}\right]}\right), \quad k=1,2,3,
\end{aligned}
$$




$$
\begin{aligned}
x_{n+1}= & z_{n}-\left(1-\frac{f\left(z_{n}\right)}{f\left(w_{n}\right)}\right)^{-1} \\
& \times\left(1-\frac{f\left(y_{n}\right)^{3}}{f\left(w_{n}\right)^{2} f\left(x_{n}\right)}\right)\left(\frac{f\left[x_{n}, y_{n}\right] f\left(z_{n}\right)}{f\left[y_{n}, z_{n}\right] f\left[x_{n}, z_{n}\right]}\right),
\end{aligned}
$$

where $\phi_{k}$ for $k=1,2,3$ is defined by (2), (3), and (4), respectively.

\section{Convergence Theorems}

Theorem 1. Let the function $f(x)$ be sufficiently differentiable in a neighborhood of its simple zero $\alpha$. If an initial approximation $x_{0}$ is sufficiently close to $\alpha$ and the parameter $\beta_{n}$ in (12) is recursively calculated by the forms given in (6)-(8), where $\phi_{k}$ for $k=1$ is defined by (2), then the R-order of convergence of the Steffensen-like method with memory (12) with the corresponding expressions (6)-(8) of $\beta_{n}$ is at least $10.7202,11$, and 11.2915, respectively.

Proof. Let $\left\{x_{n}\right\}$ be a sequence of approximations generated by an iterative method with memory (12). If this sequence converges to the zero $\alpha$ of $f$ with the $R$-order $(\geq r)$ of (12), then we write [11]

$$
e_{n+1} \sim D_{n, r} e_{n}^{r}, \quad e_{n}=x_{n}-\alpha,
$$

where $D_{n, r}$ tends to the asymptotic error constant $D_{r}$ of (12) when $n \rightarrow \infty$. Therefore,

$$
e_{n+1} \sim D_{n, r}\left(D_{n-1, r} e_{n-1}^{r}\right)^{r}=D_{n, r} D_{n-1, r}^{r} e_{n-1}^{r^{2}} .
$$

Assume that the iterative sequences $\left\{w_{n}\right\},\left\{y_{n}\right\}$, and $\left\{z_{n}\right\}$ have the $R$-orders $q$, $p$, and $s$, respectively; then, bearing in mind (13), we obtain

$$
\begin{aligned}
& \bar{e}_{n} \sim D_{n, q} e_{n}^{q}=D_{n, q}\left(D_{n-1, r} e_{n-1}^{r}\right)^{q}=D_{n, q} D_{n-1, r}^{q} e_{n-1}^{r q}, \\
& \tilde{e}_{n} \sim D_{n, p} e_{n}^{p}=D_{n, p}\left(D_{n-1, r} e_{n-1}^{r}\right)^{p}=D_{n, p} D_{n-1, r}^{p} e_{n-1}^{r p}, \\
& \hat{e}_{n} \sim D_{n, s} e_{n}^{s}=D_{n, s}\left(D_{n-1, r} e_{n-1}^{r}\right)^{s}=D_{n, s} D_{n-1, r}^{s} e_{n-1}^{r s} .
\end{aligned}
$$

Let $\phi_{k}$ for $k=1$ be defined by (2). We now obtain the order of convergence of the methods with memory (12), where $\beta_{n}$ is calculated from (6). The error relations with the selfaccelerating parameter $\beta_{n}$ for (12) are in what follows (where $\left.c_{i}=f^{(i)}(\alpha) /\left(i ! f^{\prime}(\alpha)\right), i=2,3, \ldots\right)$ :

$$
\begin{gathered}
\bar{e}_{n}=w_{n}-\alpha \sim\left(1+\beta_{n} f^{\prime}(\alpha)\right) e_{n}, \\
\widetilde{e}_{n}=y_{n}-\alpha \sim c_{2}\left(1+\beta_{n} f^{\prime}(\alpha)\right) e_{n}^{2}, \\
\widehat{e}_{n}=z_{n}-\alpha \sim c_{n, 4}\left(1+\beta_{n} f^{\prime}(\alpha)\right)^{2} e_{n}^{4}, \\
e_{n+1}=x_{n+1}-\alpha \sim c_{n, 8}\left(1+\beta_{n} f^{\prime}(\alpha)\right)^{3} e_{n}^{8} .
\end{gathered}
$$

In order to find the error relation for (12), we need to find the expression for $1+\beta_{n} f^{\prime}(\alpha)$. Using a symbolic software such as Mathematica 8 with the use of (6), we attain that

$$
1+\beta_{n} f^{\prime}(\alpha) \sim c_{4} e_{n-1} \widetilde{\mathrm{e}}_{n-1} \widehat{e}_{n-1} .
$$

According to (13), (16), (17), (19), and (22), we obtain

$$
\begin{aligned}
\widetilde{e}_{n} & \sim c_{2} c_{4} e_{n-1} \widetilde{e}_{n-1} \widehat{e}_{n-1} e_{n}^{2} \\
& \sim c_{2} c_{4} e_{n-1} D_{n-1, p} e_{n-1}^{p} D_{n-1, s} e_{n-1}^{s}\left(D_{n-1, r} e_{n-1}^{r}\right)^{2} \\
& \sim c_{2} c_{4} D_{n-1, p} D_{n-1, s} D_{n-1, r}^{2} e_{n-1}^{1+p+s+2 r} .
\end{aligned}
$$

Similarly, by (13), (16), (17), (20), and (22), we can write

$$
\begin{aligned}
\widehat{e}_{n} & \sim c_{n, 4}\left(c_{4} e_{n-1} \widetilde{e}_{n-1} \widehat{e}_{n-1}\right)^{2} e_{n}^{4} \\
& \sim c_{n, 4}\left(c_{4} e_{n-1} D_{n-1, p} e_{n-1}^{p} D_{n-1, s} e_{n-1}^{s}\right)^{2}\left(D_{n-1, r} e_{n-1}^{r}\right)^{4} \\
& \sim c_{n, 4} c_{4}^{2} e_{n-1}^{2} D_{n-1, p}^{2} e_{n-1}^{2 p} D_{n-1, s}^{2} e_{n-1}^{2 s} D_{n-1, r}^{4} e_{n-1}^{4 r} \\
& \sim c_{n, 4} c_{4}^{2} D_{n-1, p}^{2} D_{n-1, s}^{2} D_{n-1, r}^{4} e_{n-1}^{2+2 p+2 s+4 r} .
\end{aligned}
$$

Combining (13), (16), (17), (21), and (22) yields

$$
\begin{aligned}
e_{n+1} & \sim c_{n, 8}\left(c_{4} e_{n-1} \widetilde{e}_{n-1} \widehat{e}_{n-1}\right)^{3} e_{n}^{8} \\
& \sim c_{n, 8}\left(c_{4} e_{n-1} D_{n-1, p} e_{n-1}^{p} D_{n-1, s} e_{n-1}^{s}\right)^{3}\left(D_{n-1, r} e_{n-1}^{r}\right)^{8} \\
& \sim c_{n, 8} c_{4}^{3} e_{n-1}^{3} D_{n-1, p}^{3} e_{n-1}^{3 p} D_{n-1, s}^{3} e_{n-1}^{3 s} D_{n-1, r}^{8} e_{n-1}^{8 r} \\
& \sim c_{n, 8} c_{4}^{3} D_{n-1, p}^{3} D_{n-1, s}^{3} D_{n-1, r}^{8} e_{n-1}^{3+3 p+3 s+8 r} .
\end{aligned}
$$

Equating the exponents of the error $e_{n-1}$ in pairs of relations (19) and (23), (20) and (24), and then (21) and (25), we arrive at the following system of equations:

$$
\begin{gathered}
r p-1-p-s-2 r=0, \\
r s-2-2 p-2 s-4 r=0 \\
r^{2}-3-3 p-3 s-8 r=0 .
\end{gathered}
$$

Positive solution of this system is $p=(1 / 6)(7+\sqrt{109}), s=$ $(1 / 3)(7+\sqrt{109})$, and $r=(1 / 2)(11+\sqrt{109})=10.7202$. Therefore, the $R$-order of the methods with memory (12), when $\beta_{n}$ is calculated by (6), is at least 10.7202 .

Now, using a symbolic software such as Mathematica 8 with the use of (7), we attain that

$$
1+\beta_{n} f^{\prime}(\alpha) \sim c_{4} \bar{e}_{n-1} \widetilde{e}_{n-1} \widehat{e}_{n-1} .
$$

Combining (13), (15), (16), (17), (18), and (27), we obtain

$$
\begin{aligned}
\bar{e}_{n} & \sim c_{4} \bar{e}_{n-1} \widetilde{e}_{n-1} \widehat{e}_{n-1} e_{n} \\
& \sim c_{4} D_{n-1, q} e_{n-1}^{q} D_{n-1, p} e_{n-1}^{p} D_{n-1, s} e_{n-1}^{s} D_{n-1, r} e_{n-1}^{r} \\
& \sim c_{4} D_{n-1, q} D_{n-1, p} D_{n-1, s} D_{n-1, r} e_{n-1}^{q+p+s+r} .
\end{aligned}
$$


In the similar way, we find the following error relations:

$$
\begin{aligned}
\widetilde{e}_{n} & \sim c_{2} c_{4} \bar{e}_{n-1} \tilde{e}_{n-1} \widehat{e}_{n-1} e_{n}^{2} \\
& \sim c_{2} c_{4} D_{n-1, q} e_{n-1}^{q} D_{n-1, p} e_{n-1}^{p} D_{n-1, s} e_{n-1}^{s}\left(D_{n-1, r} e_{n-1}^{r}\right)^{2} \\
& \sim c_{2} c_{4} D_{n-1, q} D_{n-1, p} D_{n-1, s} D_{n-1, r}^{2} e_{n-1}^{q+p+s+2 r}, \\
\widehat{e}_{n} & \sim c_{n, 4}\left(c_{4} \bar{e}_{n-1} \widetilde{e}_{n-1} \widehat{e}_{n-1}\right)^{2} e_{n}^{4} \\
& \sim c_{n, 4}\left(c_{4} D_{n-1, q} e_{n-1}^{q} D_{n-1, p} e_{n-1}^{p} D_{n-1, s} e_{n-1}^{s}\right)^{2}\left(D_{n-1, r} e_{n-1}^{r}\right)^{4} \\
& \sim c_{n, 4} c_{4}^{2} D_{n-1, q}^{2} e_{n-1}^{2 q} D_{n-1, p}^{2} e_{n-1}^{2 p} D_{n-1, s}^{2} e_{n-1}^{2 s} D_{n-1, r}^{4} e_{n-1}^{4 r} \\
& \sim c_{n, 4} c_{4}^{2} D_{n-1, q}^{2} D_{n-1, p}^{2} D_{n-1, s}^{2} D_{n-1, r}^{4} e_{n-1}^{2 q+2 p+2 s+4 r}, \\
e_{n+1} & \sim c_{n, 8}\left(c_{4} \bar{e}_{n-1} \tilde{e}_{n-1} \widehat{e}_{n-1}\right)^{3} e_{n}^{8} \\
& \sim c_{n, 8}\left(c_{4} D_{n-1, q} e_{n-1}^{q} D_{n-1, p} e_{n-1}^{p} D_{n-1, s} e_{n-1}^{s}\right)^{3}\left(D_{n-1, r} e_{n-1}^{r}\right)^{8} \\
& \sim c_{n, 8} c_{4}^{3} D_{n-1, q}^{3} e_{n-1}^{3 q} D_{n-1, p}^{3} e_{n-1}^{3 p} D_{n-1, s}^{3} e_{n-1}^{3 s} D_{n-1, r}^{8} e_{n-1}^{8 r} \\
& \sim c_{n, 8} c_{4}^{3} D_{n-1, q}^{3} D_{n-1, p}^{3} D_{n-1, s}^{3} D_{n-1, r}^{8} e_{n-1}^{3 q+3 p+3 s+8 r} .
\end{aligned}
$$

Comparing the exponents of $e_{n-1}$ on the right hand sides of (18) and (28), (19) and (29), (20) and (30), and then (21) and (31), we arrive at the following system of equations:

$$
\begin{gathered}
r q-q-p-s-r=0, \\
r p-q-p-s-2 r=0, \\
r s-2 q-2 p-2 s-4 r=0, \\
r^{2}-3 q-3 p-3 s-8 r=0 .
\end{gathered}
$$

Positive solution of this system is $q=2, p=3, s=6$, and $r=11$. Therefore, the $R$-order of the methods with memory (12), when $\beta_{n}$ is calculated by (7), is at least 11 .

Using a symbolic software such as Mathematica 8 with the use of (8), we attain that

$$
1+\beta_{n} f^{\prime}(\alpha) \sim c_{5} e_{n-1} \bar{e}_{n-1} \widetilde{e}_{n-1} \widehat{e}_{n-1} .
$$

Using (33) and previously derived relations, we obtain the following error relations for the intermediate approximations:

$$
\begin{aligned}
\bar{e}_{n} & \sim c_{5} e_{n-1} \bar{e}_{n-1} \widetilde{e}_{n-1} \widehat{e}_{n-1} e_{n} \\
& \sim c_{5} e_{n-1} D_{n-1, q} e_{n-1}^{q} D_{n-1, p} e_{n-1}^{p} D_{n-1, s} e_{n-1}^{s} D_{n-1, r} e_{n-1}^{r} \\
& \sim c_{5} D_{n-1, q} D_{n-1, p} D_{n-1, s} D_{n-1, r} e_{n-1}^{1+q+p+s+r},
\end{aligned}
$$

$$
\begin{aligned}
\widetilde{e}_{n} & \sim c_{2} c_{5} e_{n-1} \bar{e}_{n-1} \tilde{e}_{n-1} \widehat{e}_{n-1} e_{n}^{2} \\
& \sim c_{2} c_{5} e_{n-1} D_{n-1, q} e_{n-1}^{q} D_{n-1, p} e_{n-1}^{p} D_{n-1, s} e_{n-1}^{s}\left(D_{n-1, r} e_{n-1}^{r}\right)^{2} \\
& \sim c_{2} c_{5} D_{n-1, q} D_{n-1, p} D_{n-1, s} D_{n-1, r}^{2} e_{n-1}^{1+q+p+s+2 r}, \\
\widehat{e}_{n} & \sim c_{n, 4}\left(c_{5} e_{n-1} \bar{e}_{n-1} \widetilde{e}_{n-1} \widehat{e}_{n-1}\right)^{2} e_{n}^{4} \\
& \sim c_{n, 4}\left(c_{5} e_{n-1} D_{n-1, q} e_{n-1}^{q} D_{n-1, p} e_{n-1}^{p} D_{n-1, s} e_{n-1}^{s}\right)^{2}\left(D_{n-1, r} e_{n-1}^{r}\right)^{4} \\
& \sim c_{n, 4} c_{5}^{2} e_{n-1}^{2} D_{n-1, q}^{2} e_{n-1}^{2 q} D_{n-1, p}^{2} e_{n-1}^{2 p} D_{n-1, s}^{2} e_{n-1}^{2 s} D_{n-1, r}^{4} e_{n-1}^{4 r} \\
& \sim c_{n, 4} c_{5}^{2} D_{n-1, q}^{2} D_{n-1, p}^{2} D_{n-1, s}^{2} D_{n-1, r}^{4} e_{n-1}^{2+2 q+2 p+2 s+4 r} .
\end{aligned}
$$

In the similar fashion, we find the final error relation (21) which is given by

$$
\begin{aligned}
e_{n+1} \sim & c_{n, 8}\left(c_{5} e_{n-1} \bar{e}_{n-1} \widetilde{e}_{n-1} \widehat{e}_{n-1}\right)^{3} e_{n}^{8} \\
\sim & c_{n, 8}\left(c_{5} e_{n-1} D_{n-1, q} e_{n-1}^{q} D_{n-1, p} e_{n-1}^{p} D_{n-1, s} e_{n-1}^{s}\right)^{3} \\
& \times\left(D_{n-1, r} e_{n-1}^{r}\right)^{8} \\
\sim & c_{n, 8} c_{5}^{3} e_{n-1}^{3} D_{n-1, q}^{3} e_{n-1}^{3 q} D_{n-1, p}^{3} e_{n-1}^{3 p} D_{n-1, s}^{3} e_{n-1}^{3 s} D_{n-1, r}^{8} e_{n-1}^{8 r} \\
\sim & c_{n, 8} c_{5}^{3} D_{n-1, q}^{3} D_{n-1, p}^{3} D_{n-1, s}^{3} D_{n-1, r}^{8} e_{n-1}^{3+3 q+3 p+3 s+8 r} .
\end{aligned}
$$

Comparing the exponents of $e_{n-1}$ on the right hand sides of (18) and (34), (19) and (35), (20) and (36), and then (21) and (37), we arrive at the following system of equations:

$$
\begin{gathered}
r q-q-p-s-r-1=0, \\
r p-q-p-s-2 r-1=0, \\
r s-2 q-2 p-2 s-4 r-2=0, \\
r^{2}-3 q-3 p-3 s-8 r-3=0 .
\end{gathered}
$$

Positive solution of this system is $q=(1 / 3)(1+2 \sqrt{7}), p=$ $(2 / 3)(2+\sqrt{7}), s=(4 / 3)(2+\sqrt{7})$, and $r=2(3+\sqrt{7})=11.2915$. Therefore, the $R$-order of the methods with memory (12), when $\beta_{n}$ is calculated by (8), is at least 11.2915.

Theorem 2. Let the function $f(x)$ be sufficiently differentiable in a neighborhood of its simple zero $\alpha$. If an initial approximation $x_{0}$ is sufficiently close to $\alpha$ and the parameter $\beta_{n}$ in (12) is recursively calculated by the forms given in (6)-(8), where $\phi_{k}$ for $k=2$ is defined by (3), then the R-order of convergence of the Steffensen-like method with memory (12) with the corresponding expressions (6)-(8) of $\beta_{n}$ is at least 10, 10.2426, and 10.4721, respectively.

Proof. The proof of this theorem is similar to the proof of Theorem 1; hence, it is omitted. 
TABLE 1: Families of three-point methods with memory, for example, $f_{1}(x)$.

\begin{tabular}{rcccc}
\hline Methods & $\left|x_{1}-\alpha\right|$ & $\left|x_{2}-\alpha\right|$ & $\left|x_{3}-\alpha\right|$ & $r_{c}$ \\
\hline$\phi_{1}$ & & & & \\
$\quad(12),(6)$ & $0.665 e-4$ & $0.186 e-43$ & $0.374 e-468$ & 10.737 \\
$\quad(12),(7)$ & $0.665 e-4$ & $0.718 e-44$ & $0.592 e-484$ & 11.011 \\
$\quad(12),(8)$ & $0.665 e-4$ & $0.471 e-44$ & $0.321 e-487$ & 11.038 \\
$\phi_{2}$ & & & & \\
$\quad(12),(6)$ & $0.665 e-4$ & $0.468 e-40$ & $0.800 e-402$ & 10.007 \\
$\quad(12),(7)$ & $0.665 e-4$ & $0.231 e-40$ & $0.996 e-406$ & 10.021 \\
$\quad(12),(8)$ & $0.665 e-4$ & $0.276 e-40$ & $0.579 e-405$ & 10.023 \\
$\phi_{3}$ & & & & \\
(12), (6) & $0.665 e-4$ & $0.186 e-43$ & $0.374 e-468$ & 10.737 \\
(12), (7) & $0.665 e-4$ & $0.718 e-44$ & $0.592 e-484$ & 11.011 \\
(12), (8) & $0.665 e-4$ & $0.471 e-44$ & $0.321 e-487$ & 11.038 \\
\hline
\end{tabular}

TABLE 2: Families of three-point methods with memory, for example, $f_{2}(x)$.

\begin{tabular}{rcccc}
\hline Methods & $\left|x_{1}-\alpha\right|$ & $\left|x_{2}-\alpha\right|$ & $\left|x_{3}-\alpha\right|$ & $r_{c}$ \\
\hline$\phi_{1}$ & & & & \\
(12), (6) & $0.104 e-3$ & $0.127 e-39$ & $0.868 e-425$ & 10.725 \\
(12), (7) & $0.104 e-3$ & $0.116 e-39$ & $0.419 e-436$ & 11.027 \\
$\quad(12),(8)$ & $0.104 e-3$ & $0.261 e-40$ & $0.259 e-443$ & 11.010 \\
$\phi_{2}$ & & & & \\
$\quad(12),(6)$ & $0.106 e-3$ & $0.499 e-36$ & $0.142 e-360$ & 10.040 \\
$\quad(12),(7)$ & $0.106 e-3$ & $0.476 e-36$ & $0.507 e-360$ & 10.016 \\
(12), (8) & $0.106 e-3$ & $0.223 e-36$ & $0.254 e-363$ & 10.005 \\
$\phi_{3} \quad$ & & & & \\
(12), (6) & $0.104 e-3$ & $0.127 e-39$ & $0.868 e-425$ & 10.725 \\
(12), (7) & $0.104 e-3$ & $0.116 e-39$ & $0.419 e-436$ & 11.027 \\
(12), (8) & $0.104 e-3$ & $0.261 e-40$ & $0.259 e-443$ & 11.010 \\
\hline
\end{tabular}

Theorem 3. Let the function $f(x)$ be sufficiently differentiable in a neighborhood of its simple zero $\alpha$. If an initial approximation $x_{0}$ is sufficiently close to $\alpha$ and the parameter $\beta_{n}$ in (12) is recursively calculated by the forms given in (6)-(8), where $\phi_{k}$ for $k=3$ is defined by (4), then the R-order of convergence of the Steffensen-like method with memory (12) with the corresponding expressions (6)-(8) of $\beta_{n}$ is at least 10.7202, 11, and 11.2915, respectively.

Proof. The proof of this theorem is similar to the proof of Theorem 1; hence, it is omitted.

\section{Numerical Results}

In this section, we demonstrate the convergence behavior of the methods with memory (12), where $\beta_{n}$ is calculated by one of formulas (6)-(8) and $\phi_{k}$ for $k=1,2,3$ is defined by (2)(4), respectively. Numerical computations reported here have been carried out in a Mathematica 8.0 environment. Tables 1 and 2 show the difference of the root $\alpha$ and the approximation $x_{n}$ to $\alpha$, where $\alpha$ is the exact root computed with 800 significant digits (Digits $:=800$ ). To check the theoretical order of convergence, we calculate the computational order of convergence $r_{c}$ using the following formula [14]:

$$
r_{c} \approx \frac{\log \left|f\left(x_{n}\right) / f\left(x_{n-1}\right)\right|}{\log \left|f\left(x_{n-1}\right) / f\left(x_{n-2}\right)\right|}
$$

taking into consideration the last three approximations in the iterative process. We use the following examples (selected from [11]):

$$
\begin{aligned}
f_{1}(x)= & e^{x^{2}+x \cos x-1} \sin \pi x+x \log (x \sin x+1), \\
& \alpha=0, \quad x_{0}=0.6, \quad \gamma_{0}=-0.1, \\
f_{2}(x)= & \log \left(x^{2}-2 x+2\right)+e^{x^{2}-5 x+4} \sin (x-1), \\
& \alpha=1, \quad x_{0}=1.35, \quad \gamma_{0}=-0.1 .
\end{aligned}
$$

It is obvious from the tables that recursive calculation by the Newton interpolation (8) gives the best results.

\section{Conclusion}

In this paper, Newton's interpolatory polynomials of the third and fourth degrees are applied for constructing considerably faster methods employing information from the current and previous iteration without any additional evaluations of the function. $11.2915^{1 / 4}=1.83311$ has been obtained as the highest possible computational efficiency index for the new methods with memory. The efficacy of the methods is tested on a number of numerical examples. The results show that this method is very useful to find an acceptable approximation of the exact solution of nonlinear equations.

\section{Conflict of Interests}

The author declares that there is no conflict of interests regarding the publication of this paper.

\section{Acknowledgment}

The author is grateful to the referees for their comments and suggestions that helped to improve the paper.

\section{References}

[1] J. F. Traub, Iterative Methods for the Solution of Equations, Prentice-Hall, New Jersey, NJ, USA, 1964.

[2] J. F. Steffensen, "Remarks on iteration," Skandinavisk Aktuarietidskrift, vol. 16, pp. 64-72, 1933.

[3] H. T. Kung and J. F. Traub, "Optimal order of one-point and multipoint iteration," Journal of the Association for Computing Machinery, vol. 21, pp. 643-651, 1974.

[4] F. Soleymani and S. Shateyi, "Some iterative methods free from derivatives and their basins of attraction for nonlinear equations," Discrete Dynamics in Nature and Society, vol. 2013, Article ID 301718, 10 pages, 2013.

[5] F. Soleymani, S. Karimi Vanani, and M. Jamali Paghaleh, "A class of three-step derivative-free root solvers with optimal 
convergence order," Journal of Applied Mathematics, vol. 2012, Article ID 568740, 15 pages, 2012.

[6] F. Soleymani, "Some optimal iterative methods and their with memory variants," Journal of the Egyptian Mathematical Society, vol. 21, no. 2, pp. 133-141, 2013.

[7] F. Soleymani and V. Hosseinabadi, "New third- and sixth-order derivative-free techniques for nonlinear equations," Journal of Mathematics Research, vol. 3, 2011.

[8] T. Eftekhari, "A new sixth-order Steffensen-type iterative method for solving nonlinear equations," International Journal of Analysis, vol. 2014, Article ID 685796, 5 pages, 2014.

[9] M. Dehghan and M. Hajarian, "Some derivative free quadratic and cubic convergence iterative formulas for solving nonlinear equations," Computational \& Applied Mathematics, vol. 29, no. 1, pp. 19-30, 2010.

[10] A. Cordero, J. L. Hueso, E. Martínez, and J. R. Torregrosa, "Steffensen type methods for solving nonlinear equations," Journal of Computational and Applied Mathematics, vol. 236, no. 12, pp. 3058-3064, 2012.

[11] J. Džunić, M. S. Petković, and L. D. Petković, “Three-point methods with and without memory for solving nonlinear equations," Applied Mathematics and Computation, vol. 218, no. 9, pp. 4917-4927, 2012.

[12] R. Thukral, "A family of three-point derivative-free methods of eighth-order for solving nonlinear equations," Journal of Modern Methods in Numerical Mathematics, vol. 3, no. 2, pp. 11-21, 2012.

[13] J. R. Sharma, R. K. Guha, and P. Gupta, "Some efficient derivative free methods with memory for solving nonlinear equations," Applied Mathematics and Computation, vol. 219, no. 2, pp. 699-707, 2012.

[14] M. S. Petković, "Remarks on "On a general class of multipoint root-finding methods of high computational efficiency"', SIAM Journal on Numerical Analysis, vol. 49, no. 3, pp. 1317-1319, 2011. 


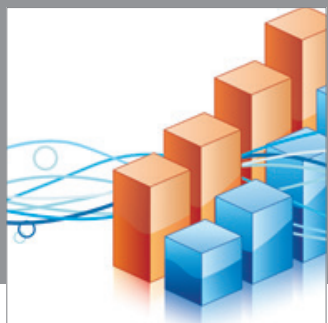

Advances in

Operations Research

mansans

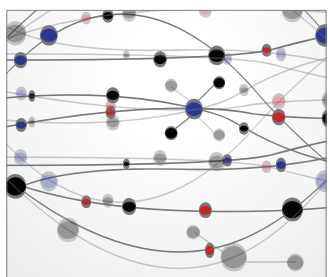

The Scientific World Journal
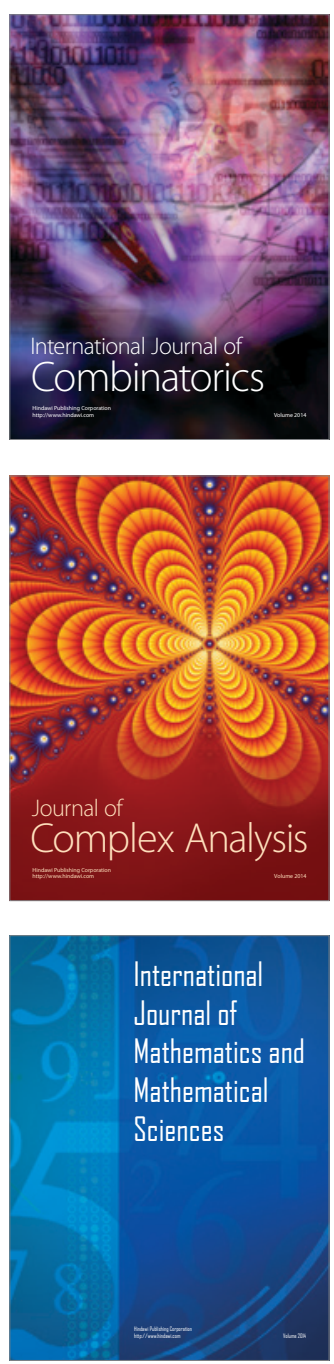
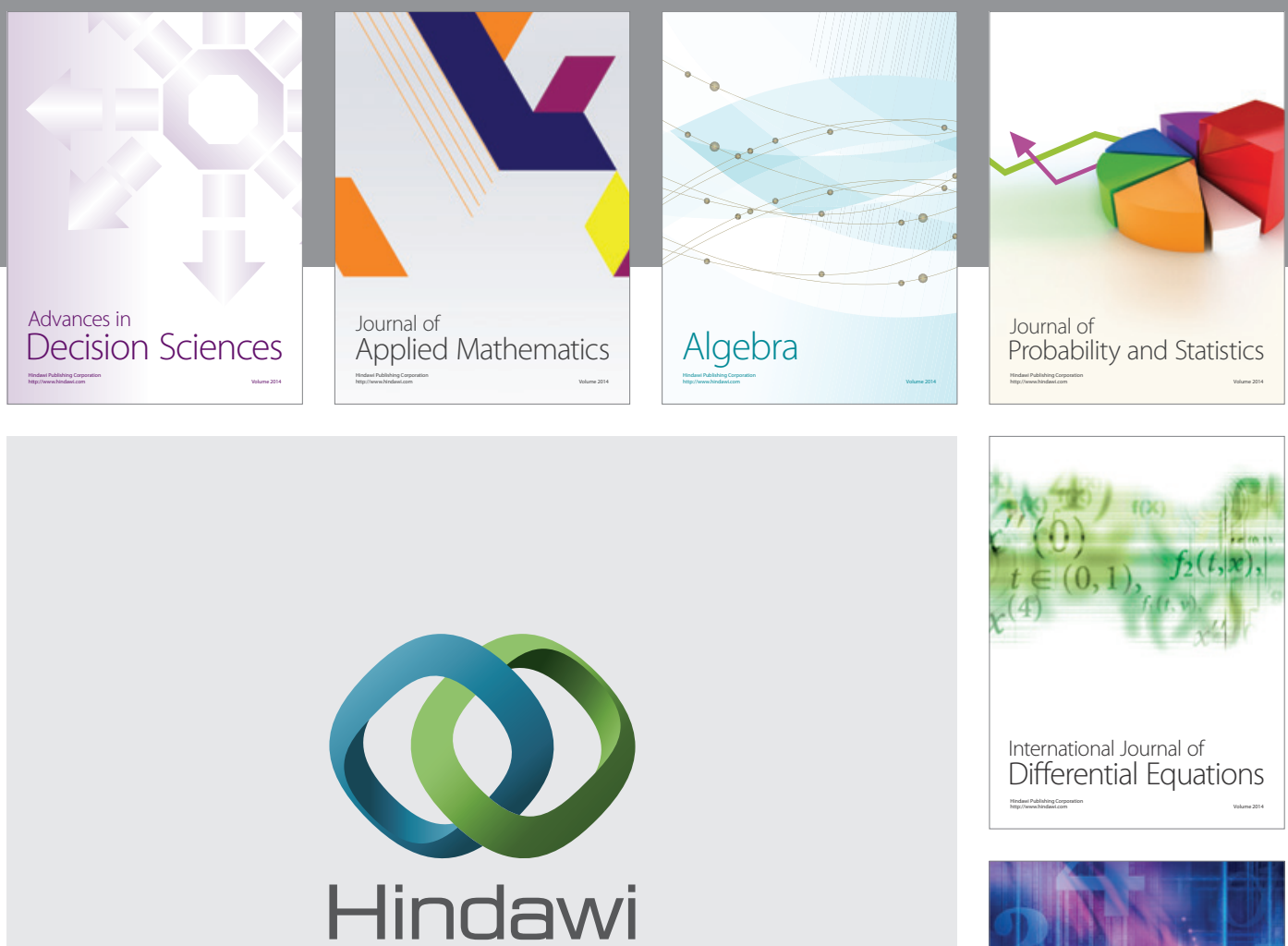

Submit your manuscripts at http://www.hindawi.com
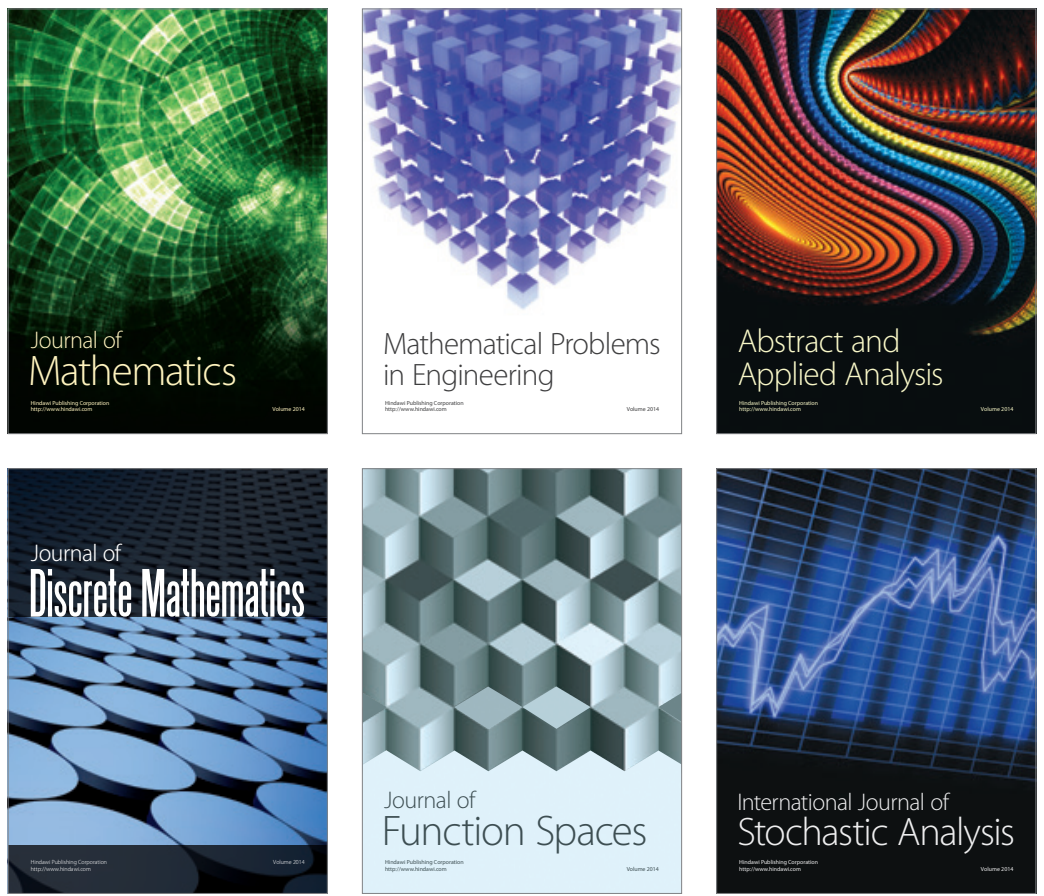

Journal of

Function Spaces

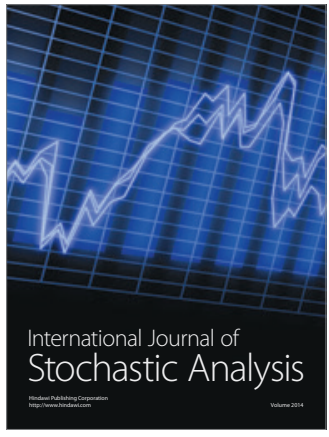

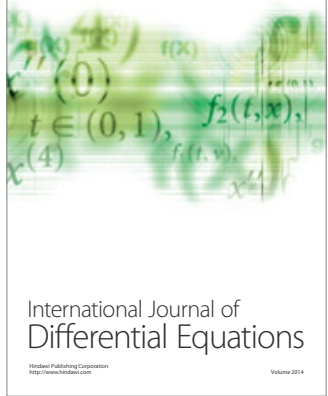
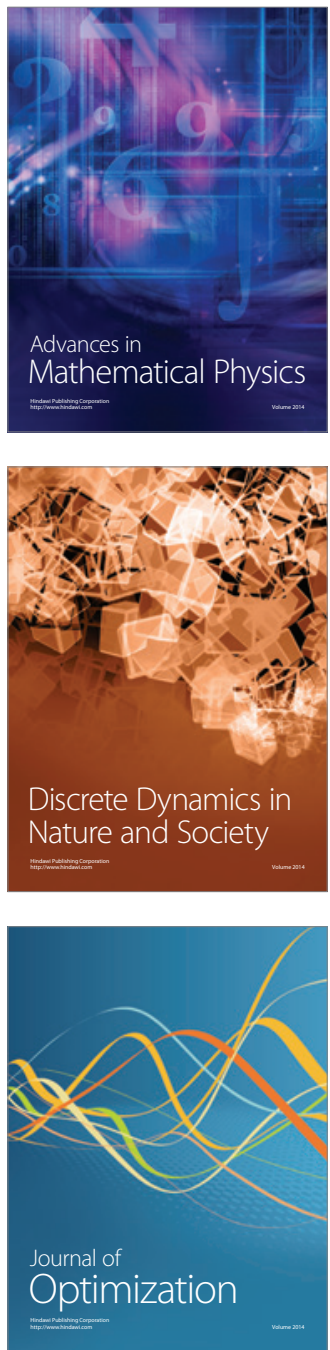